\title{
FUNCTIONS WHICH HAVE HARMONIC SUPPORT
}

\author{
ALAN WILSON
}

In $[1$, p. 302] Johnson makes the

Definition. A function v(z) real-valued in a domain $D$ of the complex $z$ plane has harmonic support [belongs to the class (hs)] in $D$ if (I) $v$ is bounded on each compact subset of $D$ and (II) for each $z_{0}$ in $D$ there exists $h$ harmonic and single-valued in $D$ such that $v\left(z_{0}\right)=h\left(z_{0}\right)$ and $h \leqq v$ in $D$.

As stated in [1], it follows immediately from the definition that a function of ( $h s)$ in $D$ is subharmonic in $D$. Indeed the same conclusion follows if in (I) "bounded" is replaced with "bounded above." We ask whether (I) can be dispensed with altogether. That is, Does (II) alone imply subharmonicity? Henceforth let $D$ denote the open plane and $\gamma$ the non-negative real axis. We prove:

There exists a function satisfying (II) in $D$ which is subharmonic in $D-\gamma$ and which is unbounded in every neighborhood of every point of $\gamma$.

Since a subharmonic function must be bounded above on each compact set, the answer to our question is no.

As shown in [2, pp. 122-123], there exists a nonconstant entire function $F(z)$ such that $F \rightarrow 0$ along each ray and, for any fixed $\sigma>0, F \rightarrow 0$ uniformly as $z \rightarrow \infty$ in the sector $W[\sigma]: \sigma \leqq \arg z \leqq 2 \pi-\sigma$. Let $\left\{w_{n}\right\}$ be a sequence of nonzero complex numbers tending to infinity such that $F\left(w_{n}\right) \neq 0, F\left(w_{n}\right) \rightarrow \infty$. Consider the sequence of entire functions $f_{n}(z)=F\left(\left|w_{n}\right|{ }^{2} z\right)\left|F\left(w_{n}\right)\right|^{-1 / 2}$ and let $v(z)=\sup _{n}\left|f_{n}(z)\right|$.

A. A compact set $\Delta \subset D-\gamma$ is also $\subset W[\sigma]$ for a sufficiently small positive $\sigma$. Hence, $f_{n}(z) \rightarrow 0$ uniformly on $\Delta$ and in particular $\left\{f_{n}(z)\right\}$ is uniformly bounded on $\Delta$. Therefore $v$ is bounded on $\Delta$. Since $\Delta$ is arbitrary, $v$ satisfies (I) in $D-\gamma$.

B. For each fixed $z_{0}$ in $D$ we have $f_{n}\left(z_{0}\right) \rightarrow 0$, so that there is an integer $m\left(z_{0}\right)=m$ for which $\left|f_{m}\left(z_{0}\right)\right|=\sup _{n}\left|f_{n}\left(z_{0}\right)\right|=v\left(z_{0}\right)$. According to Johnson [1, p. 320], $\left|f_{m}\right|$ has an (entire) harmonic support function at $z_{0}$. Since $\left|f_{m}\right| \leqq v$, we conclude that $v$ has a harmonic support function at $z_{0}$. Since $z_{0}$ is arbitrary, $v$ satisfies (II) in $D .{ }^{1}$

Combining the results of $\mathrm{A}$ and $\mathrm{B}$, we see that $v$ belongs to $(h s)$ in $D-\gamma$ and is therefore subharmonic there.

Received by the editors November 22, 1961 and, in revised form, November 28, 1961.

1 The reasoning in B was suggested in part by a remark of W. K. Hayman in private conversation. 
C. Let $z_{n}=w_{n}\left|w_{n}\right|^{-2}$. Then $z_{n} \rightarrow 0$ and $v\left(z_{n}\right) \geqq\left|f_{n}\left(z_{n}\right)\right|=\left|F\left(w_{n}\right)\right|^{1 / 2}$ $\rightarrow \infty$. Thus $v$ is unbounded in every neighborhood of the origin.

Suppose that $v$ is bounded $\left(v \leqq M_{1}\right)$ on $\left|z-x_{0}\right| \leqq r$, where $x_{0}>0$ and $0<r<x_{0}$. Let $\sigma_{0}=\sin ^{-1} r / x_{0}$. Since $F \rightarrow 0$ uniformly as $z \rightarrow \infty$ in $W\left[\sigma_{0}\right]$, there is a finite $M_{2}$ such that $|F(z)| \leqq M_{2}$ on $W\left[\sigma_{0}\right]$. Choose $N\left(\sigma_{0}\right)=N$ so that for $n \geqq N$ we have $\left|F\left(w_{n}\right)\right|>M_{2}$ and $\left|w_{n}\right| x_{0}>1$. It follows on the one hand that for $n \geqq N, w_{n}$ is in the complement of $W\left[\sigma_{0}\right]$, and on the other hand that $w_{n}$ is in the circle $|z|<\left|w_{n}\right|^{2} x_{0}$. By hypothesis $|F(z)| \leqq M_{1}\left|F\left(w_{n}\right)\right|^{1 / 2}$ on $|z-| w_{n}\left|{ }^{2} x_{0}\right| \leqq r\left|w_{n}\right|^{2}$, and in particular the inequality holds on the arc of $|z|=\left|w_{n}\right|^{2} x_{0}$ contained in the closure of the complement of $W\left[\sigma_{0}\right]$. By the definition of $\sigma_{0}$ and the maximum principle we have $\left|F\left(w_{n}\right)\right| \leqq \max \left[M_{1}\left|F\left(w_{n}\right)\right|^{1 / 2}, M_{2}\right]$ for $n \geqq N$. This is evidently false, so $v$ is not bounded on $\left|z-x_{0}\right| \leqq r$. Since $x_{0}$ is arbitrary, $v$ is unbounded in the neighborhood of every point of $\gamma$.

We observe that the sequence of entire functions $\left\{f_{n}(z)\right\}$ is pointwise bounded in $D$ but is not uniformly bounded on any compact subset of $D$ which intersects $\gamma$. Thus the following statement, appearing in $[3$, p. 97], is false: "A pointwise bounded family of functions analytic in a domain is bounded subuniformly on each compact subset of the domain."

\section{REFERENCES}

1. G. Johnson, Jr., Functions which have harmonic support, Trans. Amer. Math. Soc. 92 (1959), 302-321.

2. E. Lindelöf, Calcul des résidus, Chelsea, New York, 1947.

3. A. Dinghas, Vorlesungen über Funktionentheorie, Springer, Berlin, 1960.

IMPERIAL COLLEGE, LONDON 